\title{
NONCONTACT DETERMination OF THERMAL DIFFUSIVITY IN BIOMATERIALS USING INFRARED IMAGING RADIOMETRY
}

\author{
Thomas E. Milner, ${ }^{\dagger}$ Dennis M. Goodman, ${ }^{\dagger}$ B. Samuel Tanenbaum, ${ }^{*}$ Bahman Anvari, ${ }^{* *}$ \\ and J. Stuart Nelson ${ }^{\S}$ \\ †University of California Irvine, Beckman Laser Institute and Medical Clinic, Irvine, California \\ 92715, and Harvey Mudd College, Department of Physics, Claremont, California 91711; ${ }^{*}$ Lawrence \\ Livermore National Laboratory, Laser Engineering Division, Livermore, California 94551, \\ *Harvey Mudd College, Department of Engineering, Claremont, California; **University of California \\ Irvine, Beckman Laser Institute and Medical Clinic, Irvine, California, and Harvey Mudd \\ College, Department of Engineering, Claremont, California; ${ }^{\S}$ University of California Irvine, Beckman \\ Laser Institute and Medical Clinic, Departments of Surgery and Dermatology, Irvine, California \\ (Paper JBO-021 received July 10, 1995; revised manuscript received Nov. 7, 1995; accepted for publication Nov. 7, 1995)
}

\begin{abstract}
A noncontact method to determine the thermal diffusivity of biomaterials is derived theoretically and demonstrated experimentally. Assuming that the initial three-dimensional temperature distribution in the biomaterial immediately following pulsed laser irradiation can be separated into lateral and longitudinal factors, a convolution equation is derived that relates a pair of infrared emission images to the lateral thermal point spread function. Pairs of infrared emission images recorded following pulsed laser irradiation are used in combination with a nonlinear least-squares algorithm to determine the lateral thermal point spread function and thus the thermal diffusivity of the biomaterial. Sequences of infrared emission images recorded following pulsed laser irradiation are used to determine thermal diffusivity $\left(0.103 \pm 0.007 \mathrm{~mm}^{2} / \mathrm{s}\right)$ of an in vitro type I hydrated collagen film. Application of the method to in vivo tissues is discussed.
\end{abstract}

Key Words radiometry; biomaterials; thermal diffusivity; collagen.

\section{INTRODUCTION}

Thermal diffusivity $(\chi)$ is an important physical parameter that determines the spatial extent of heat transport in the presence of a temperature gradient. Determination of $\chi$ in biomaterials is important for various biomedical applications. For example, the successful therapeutic outcome of many laser surgical procedures relies on selective absorption of radiation to destroy or transform targeted absorbers by thermal-induced protein denaturation; knowledge of $\chi$, therefore, is essential to understand and accurately predict the incident light dose necessary to maximize damage to the target while at the same time minimizing nonspecific injury to adjacent structures.

Determination of $\chi$ requires a technique to measure temperature (or an equivalent quantity) in response to an artificially induced thermal gradient at discrete positions in the biomaterial. In addition, a physical model of heat transfer associated with the measurement is required to interpret data and deduce thermal diffusivity. Bowman, Cravalho, and Woods ${ }^{1}$ identified three general classes of tech-

Address all correspondence to Thomas E. Milner, Beckman Laser Institute and Medical Clinic, 1002 Health Sciences Road East, Irvine, CA 92715.

Fax: (714) 824-8413. E-mail: temilner@uci.edu niques to determine thermal diffusivity in biomaterials using probes that are (1) invasive, (2) semiinvasive, and (3) noninvasive. In their characterization, a probe can either function to induce a thermal gradient (stimulator) and/or measure temperature response (detector). A primary disadvantage of invasive or semi-invasive probes is the measurement artifacts introduced by mechanical and/or thermal trauma. The introduction of novel laser sources and high-performance infrared detectors has led to the development of completely noninvasive methods to determine various thermophysical properties of biomaterials. ${ }^{2}$

We present a noncontact method provided by infrared imaging radiometry to determine thermal diffusivity of a biomaterial from radiometric temperature measurements following pulsed laser irradiation. The method is based on estimating the lateral thermal point spread function by a nonlinear least-squares algorithm using as input data pairs of recorded infrared emission images. First, the theory of the method is derived, including effects of heat diffusion and inherent limitations of the infrared focal plane array (IR-FPA) camera system. Second, infrared emission image pairs of an in vitro hydrated

1083-3668/96/\$6.00 (C) 1996 SPIE 


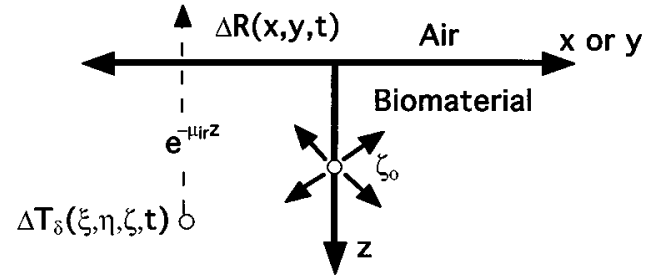

Fig. 1 Geometry assumed in the analysis to determine thermal diffusivity. $\Delta R(x, y, t)$ is the radiometric temperature increase in response to thermal excitation at coordinate position $\left(0,0, \zeta_{0}\right)$. Infrared emission due to temperature increase $\left[\Delta T_{\delta}\right]$ at position $(\xi, \eta, \zeta)$ is exponentially attenuated $\left(\mathrm{e}^{-\mu_{i r} z}\right)$ upon exit from the biomaterial.

type I collagen film are used to estimate the lateral thermal point spread function and thus determine thermal diffusivity.

\section{THEORY}

We derive an integral equation that relates the time evolution of measured infrared emission images $[\Delta M(x, y, t)]$ of a biomaterial following pulsed laser irradiation to the initial three-dimensional temperature increase $\left[\Delta T^{3-D}(x, y, z, t=0)\right]$. When lateral and longitudinal components of $\Delta T^{3-D}(x, y, z, t=0)$ are separable, a recorded infrared emission image $\left[\Delta M\left(x, y, t_{2}\right)\right]$ can be expressed as a convolution of the lateral thermal point spread function $\left[K_{r}(\Delta t\right.$ $\left.\left.=t_{2}-t_{1}\right)\right]$ and $\Delta M\left(x, y, t_{1}\right)$ at an earlier time $\left(t_{1}<t_{2}\right)$. Pairs of recorded infrared emission images in combination with a modified LevenbergMarquardt nonlinear least-squares algorithm are used to determine $K_{r}$ and thus determine thermal diffusivity of the biomaterial.

For the purpose of deriving the integral equation, we use a rectilinear coordinate system (Figure 1) and assume that a $\delta$ pulse of energy $q[J]$ is released within a semi-infinite medium (depth $z=\zeta_{0}$, time $t=0$ ) that represents a biomaterial in thermal equilibrium. We seek the space-time-dependent temperature increase $\left(\Delta T_{\delta}\right)$ by solving the bioheat equation,

$$
\begin{gathered}
\nabla^{2}\left(\Delta T_{\delta}\right)-\frac{1}{\chi} \frac{\partial\left(\Delta T_{\delta}\right)}{\partial t}-\frac{Q}{\chi} \Delta T_{\delta} \\
=\frac{q / \rho C}{\chi} \delta(t) \delta(x) \delta(y) \delta\left(z-z_{0}\right) .
\end{gathered}
$$

In Eq. (1) $Q[1 / s]$ represents the volume fraction of perfused blood per unit time and in general may depend not only on position but the biomaterial itself; $\rho C\left(\mathrm{~J} / \mathrm{m}^{3} \cdot \mathrm{K}\right)$ is the product of specific heat capacity (C) $(\mathrm{J} / \mathrm{kg} \cdot \mathrm{K})$ and density $(\rho)\left(\mathrm{kg} / \mathrm{m}^{3}\right)$; $\chi\left(\mathrm{m}^{2} / \mathrm{s}\right)$ is the thermal diffusivity of a homogeneous biomaterial.

To solve Eq. (1), we assume a Robin-type boundary condition,

$$
\left.\kappa \frac{\partial\left(\Delta T_{\delta}\right)}{\partial z}\right|_{z=0}=\left.h \Delta T_{\delta}\right|_{z=0}
$$

in which $\kappa(\mathrm{W} / \mathrm{m} \cdot \mathrm{K})$ is thermal conductivity and the heat loss coefficient, $h\left(\mathrm{~W} / \mathrm{m}^{2} \cdot \mathrm{K}\right)$, represents the combined radiative and/or convective thermal energy losses at the air-surface interface.

Solution of the bioheat equation $\left[\Delta T_{\delta}(\xi, \eta, \zeta, t)\right]$ represents the temperature increase above ambient values at position $(\xi, \eta, \zeta)$ and time $(t)$ in response to a $\delta$ pulse of energy released at position $\left(0,0, \zeta_{0}\right)$ and time $t=0$.

$$
\begin{aligned}
\Delta T_{\delta}(\xi, \eta, \zeta, t)= & \frac{(q / \rho C) e^{-Q t}}{(4 \pi \chi t)^{3 / 2}} \mathrm{e}^{-\left(\xi^{2}+\eta^{2}\right) / 4 \pi} \\
& \cdot\left\{\mathrm{e}^{-\left(\zeta-\zeta_{0}\right)^{2} / 4 \chi t}+\mathrm{e}^{-\left(\zeta+\zeta_{0}\right)^{2} / 4 \chi^{t}}\right. \\
& \left.\times\left[1-\frac{h}{\kappa} \sqrt{4 \pi \chi t} \operatorname{erfc} x(u)\right]\right\},
\end{aligned}
$$

where

$$
u=\frac{\zeta+\zeta_{0}}{2 \sqrt{\chi t}}+\frac{h}{\kappa} \sqrt{\chi t}
$$

and $\operatorname{erfc} x(u)=\exp \left(u^{2}\right) \operatorname{erfc}(u)$, where $\operatorname{erfc}(\cdot)$ is the complementary error function [1-erf(.)]. Furthermore, we have assumed that the volume fraction of perfused blood per unit time $(Q)$ is uniform over the region of interest.

$\Delta T_{\delta}$ represents a temperature increase in the biomaterial above equilibrium levels. In many biomaterials such as in vivo human skin, the equilibrium temperature distribution is not homogeneous and oftentimes exhibits a gradient normal to the surface (i.e., $d T / d z>0$ ). In these cases, our analysis remains valid if thermal excitation [represented on the righthand side of Eq. (1)] does not interfere with the normal physiological regulatory mechanisms sustaining thermal equilibrium.

Thermal excitation of the biomaterial gives rise to increased radiometric temperatures; at each position in the biomaterial we assume local thermal equilibrium is attained. In addition, we assume infrared emission is absorbed exponentially and that the infrared absorption coefficient $\left(\mu_{\mathrm{ir}}\right)$ is independent of depth and constant over relevant temperature changes (Figure 1). If the change in infrared emission at the biomaterial surface due to an infinitesimal volume of excess thermal energy (with unit temperature difference above the background level) located at position $(\xi, \eta, \zeta)$ is a linear function of $\Delta T_{\delta}$, the corresponding radiometric temperature increase is

$$
\mu_{i r} \mathrm{e}^{-\mu_{i r} \zeta} \delta(x-\xi) \delta(y-\eta)
$$

By integrating the excess thermal energy over the entire biomaterial volume, the relative increase in infrared emission is 


$$
\begin{aligned}
K_{T} \propto \mu_{i r} \int & \iint_{\xi, \eta, \zeta} \mathrm{e}^{-\mu_{i r} \zeta} \delta(x-\xi) \\
& \times \delta(y-\eta) \Delta T_{\delta}(\xi, \eta, \zeta, t) d \xi d \eta d \zeta .
\end{aligned}
$$

For a $\delta$ pulse of energy, $q$ (depth $z=\zeta_{0}$, time $t=0$ ), substituting $\Delta T_{\delta}(\xi, \eta, \zeta, t)$ [Eq. (3)] into Eq. (4b) gives

$$
\begin{aligned}
& K_{T} \propto \frac{\mathrm{e}^{-Q t}(q / \rho C)}{4 \pi \chi^{t}} \mathrm{e}^{-\left(x^{2}+y^{2}\right) / 4 \chi^{t}} \frac{\mu_{i r}}{2} \mathrm{e}^{-\xi_{0}^{2} / 4 \chi^{t}} \\
& \cdot\left\{\operatorname{erfc} x\left(u_{+}\right)+\operatorname{erfc} x\left(u_{-}\right)-\frac{2(h / \kappa)}{(h / \kappa)-\mu_{i r}}\right. \\
&\left.\quad \times\left[\operatorname{erfc} x\left(u_{+}\right)-\operatorname{erfc} x\left(u_{1}\right)\right]\right\},
\end{aligned}
$$

where

$$
u_{ \pm}=\mu_{i r} \sqrt{\chi^{t}} \pm \frac{\zeta_{0}}{2 \sqrt{\chi^{t}}}, \quad u_{1}=\frac{h}{\kappa} \sqrt{\chi^{t}}+\frac{\zeta_{0}}{2 \sqrt{\chi^{t}}} .
$$

Since $q /(\rho C)$ is proportional to the initial temperature increase at position $\left(0,0, \zeta_{0}\right), K_{T}$ is written

$$
K_{T}\left(x-\xi, y-\eta, \zeta_{0}, t\right)=K_{r}(x-\xi, y-\eta, t) \cdot K_{z}\left(\zeta_{0}, t\right),
$$

where the expression for $K_{T}$ [Eq. (7)] consists of two distinct terms [Eqs. (8a) and (8b)] that represent, respectively, heat diffusion along lateral $\left(K_{r}\right)$ and longitudinal $\left(K_{z}\right)$ axes.

$$
\begin{gathered}
K_{r}(x, y, t)=\frac{1}{4 \pi \chi t} \mathrm{e}^{-\left(x^{2}+y^{2}\right) / 4 \chi^{t}} \\
K_{z}\left(z_{0}, t\right)=\frac{\mu_{i r}}{2} \mathrm{e}^{-Q t} \mathrm{e}^{-z_{0}^{2} / 4 \chi t}\left\{\operatorname{erfc} x\left(u_{+}\right)+\operatorname{erfc} x\left(u_{-}\right)\right. \\
\left.-\frac{2(h / \kappa)}{(h / \kappa)-\mu_{i r}}\left[\operatorname{erfc} x\left(u_{+}\right)-\operatorname{erfc} x\left(u_{1}\right)\right]\right\} .
\end{gathered}
$$

The effect of lateral $\left(K_{r}\right)$ and longitudinal $\left(K_{z}\right)$ heat diffusion on the radiometric temperature increase $[\Delta R(x, y, t)]$ can be understood by writing Eq. (9) in terms of $K_{r}$ and $K_{z}$,

$$
\begin{aligned}
\Delta R(x, y, t)= & \iint_{\zeta, \eta} d \xi d \eta \cdot K_{r}(x-\xi, y-\eta, t) \\
& \cdot\left[\int_{\zeta} d \zeta \cdot K_{z}(\zeta, t) \cdot \Delta T^{3-D}(\xi, \eta, \zeta, t=0)\right] .
\end{aligned}
$$

Examination of Eq. (9) reveals that the transverse distribution of a radiometric temperature increase (i.e., $x, y$ dependence) is given by a convolution integral (in the $\xi, \eta$ plane) containing the lateral ther- mal point spread function $\left(K_{r}\right)$ which represents thermal diffusion parallel to the air-surface interface. The relative amplitude of $\Delta R(x, y, t)$ is also related to an integral (i.e., bracketed term containing $K_{z}$ ) which represents longitudinal thermal diffusion perpendicular to the air-surface interface.

Because the radiometric temperature at the biomaterial surface is not measured directly, we account for inherent limitations of the IR-FPA camera and write an infrared emission image $[\Delta M(x, y, t)]$ as a two-dimensional convolution integral in terms of $\Delta R(x, y, t)$,

$$
\Delta M(x, y, t)=\iint_{\xi, \eta} K_{c}(x-\xi, y-\eta) \cdot \Delta R(\xi, \eta, t) d \xi d \eta
$$

The camera point spread function $\left(K_{c}\right)$ includes effects of collection aperture size, lens aberrations, and finite number and size of discrete IR-FPA detector elements.

Our method for determining thermal diffusivity requires that the initial three-dimensional temperature increase $\left[\Delta T^{3-D}(x, y, z, t=0)\right]$ be separated into lateral $\left[\Delta T^{2-D}(x, y, t=0)\right]$ and longitudinal $\left[\Delta T^{1-D}(z, t=0)\right]$ factors,

$$
\begin{aligned}
\Delta T^{3-D}(x, y, x, t=0)= & \Delta T^{2-D}(x, y, t=0) \\
& \cdot \Delta T^{1-D}(z, t=0) .
\end{aligned}
$$

The separability assumption is satisfied when the initial three-dimensional temperature increase is generated by absorption of short pulsed laser radiation and either: (1) absorption of normally incident light is much stronger than scattering and thus Beer's law [i.e., $\Delta T^{3-D}(x, y, z, t=0)=\Delta T_{0}(x, y)$ $\mathrm{e}^{-\mu_{a} z}$ applies; or (2) subsurface chromophores are identically heated and confined to a layer positioned at depth $z_{0}$ [i.e., $\Delta T^{3-D}(x, y, z, t=0)$ $\left.=\Delta T_{0}(x, y) \cdot f\left(z-z_{0}\right)\right]$. The former case is realized for long wavelength laser radiation (e.g., $\mathrm{CO}_{2}$ at $\lambda=10.6 \mu \mathrm{m}$ ), which is strongly absorbed by protein and water in biomaterials. Subsequently, we analyze the latter case which applies to an in vitro system where subsurface chromophores are confined to a single layer.

Given the separability assumption [Eq. (11)], expressions for the radiometric temperature increase $[\Delta R(x, y, t)$, Eq. (9)] and camera point spread function $\left[K_{c}\right.$, Eq. (10)], the infrared emission image is,

$$
\begin{aligned}
\Delta M(x, y, t)= & \int_{\zeta} d \zeta \cdot K_{z}(\zeta, t) \cdot \Delta T^{1-D}(\zeta, t=0) \\
& \times \iint_{\xi, \eta} d \xi d \eta \cdot\left(K_{c} * K_{r}\right)(x-\xi, y-\eta) \\
& \cdot \Delta T^{2-D}(\xi, \eta, t=0) .
\end{aligned}
$$

The longitudinal diffusion integral in Eq. (12) is in- 
dependent of lateral coordinates (i.e., $x, y$ ) so that $\Delta M(x, y, t)$ is proportional [Eq. (13)] to the product of a two-dimensional convolution integral and time-dependent amplitude $[S(t)]$,

$$
\begin{aligned}
\Delta M(x, y, t)= & S(t) \cdot \iint_{\xi, \eta} d \xi d \eta \cdot\left(K_{c} * K_{r}\right)(x-\xi, y-\eta, t) \\
& \cdot \Delta T^{2-D}(\xi, \eta, t=0) .
\end{aligned}
$$

An expression that relates infrared emission images recorded at two times, $t_{1}<t_{2}$, is derived from convolution of the lateral thermal point spread function $\left[K_{r}\right.$ Eq. (8a)],

$$
K_{r}\left(t_{2}\right)=K_{r}\left(\Delta t=t_{2}-t_{1}\right) * K_{r}\left(t_{1}\right) .
$$

Since the convolution operation (i.e., $*$ ) is both commutative and associative and the camera point spread function $\left(K_{c}\right)$ is independent of time, the infrared emission image at time $t_{2}$ is

$$
\begin{aligned}
& \Delta M\left(x, y, t_{2}\right) \\
& =S\left(t_{2}\right) \cdot \iint_{\xi, \eta} d \xi d \eta \cdot K_{r}\left(x-\xi, y-\eta, t_{2}-t_{1}\right) \\
& \quad \times \iint_{\xi^{\prime}, \eta^{\prime}} d \xi^{\prime} d \eta^{\prime} \cdot\left(K_{c} * K_{r}\right)\left(\xi-\xi^{\prime}, \eta-\eta^{\prime}, t_{1}\right) \\
& \cdot \Delta T^{2-D}\left(\xi^{\prime}, \eta^{\prime}, t=0\right) .
\end{aligned}
$$

The basic working equation for our analysis is obtained by rewriting the integrand in Eq. (15) in terms of $\Delta M\left(x, y, t_{1}\right)$,

$$
\begin{aligned}
\Delta M\left(x, y, t_{2}\right)= & \frac{S\left(t_{2}\right)}{S\left(t_{1}\right)} \cdot \iint_{\xi, \eta} d \xi d \eta \cdot K_{r}\left(x-\xi, y-\eta, t_{2}-t_{1}\right) \\
& \cdot \Delta M\left(\xi, \eta, t_{1}\right) \\
= & C\left(t_{2}, t_{1}\right) \cdot K_{r}(\Delta t) * \Delta M\left(t_{1}\right) .
\end{aligned}
$$

Thus, infrared emission images recorded at any two times separated by $\Delta t=t_{2}-t_{1}$ are simply related by a convolution of the lateral thermal point spread function $\left[K_{r}(\Delta t)\right]$ and a constant $C\left(t_{2}, t_{1}\right)$. The goal of our analysis is to determine the thermal diffusivity by estimating the lateral thermal point spread function from pairs of measured infrared emission images. Because convolution of two functions simplifies to multiplication in the frequency domain, we Fourier transform $(\mathscr{T})$ the basic working equation [Eq. (16)] in the $(x, y)$ coordinate plane and determine $\chi$ by minimizing $f\left(\chi, C, x_{0}, y_{0}\right)$

$$
\begin{aligned}
f\left(\chi, C, x_{0}, y_{0}\right)= & (\mathscr{F} \Delta M)\left(k_{x}, k_{y}, t_{2}\right) \\
& -C \cdot\left(\mathscr{F} K_{r}\right)\left(k_{x}, k_{y}, \Delta t ; \chi, x_{0}, y_{0}\right) \\
& \cdot(\mathscr{F} \Delta M)\left(k_{x}, k_{y}, t_{1}\right) .
\end{aligned}
$$

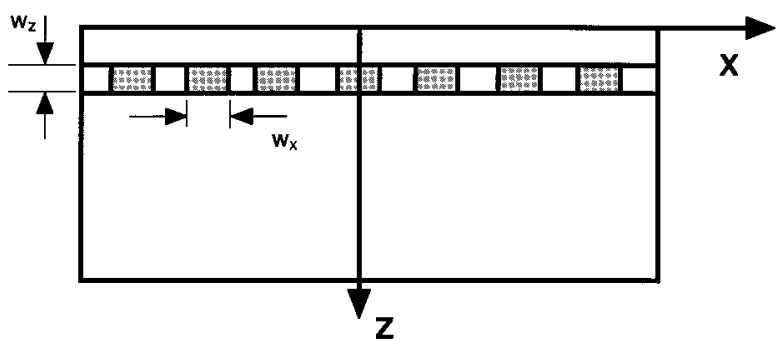

Fig. 2 Schematic diagram of the in vitro model used in experiments.

Parameters $x_{0}$ and $y_{0}$ are introduced to account for any lateral offset between image pairs that may be present in $K_{r}$. In the spatial frequency domain, Eq. (17) represents a set of nonlinear equations; the unknown parameters (i.e., $\left.\chi, C, x_{0}, y_{0}\right)$ of the lateral thermal point spread function $\left[K_{r}(\Delta t)\right]$ are determined from each independent pair of infrared emission images recorded at times separated by $\Delta t=t_{2}-t_{1}$. Mean thermal diffusivity $(\bar{\chi})$ is computed by a weighted average of $\chi$ values obtained from all pairs of independent infrared emission images recorded following pulsed laser irradiation of the biomaterial.

\section{Methods AND ReSUltS}

Experiments are described that record a time sequence of infrared emission images $[\Delta M(x, y, t)]$ in response to pulsed laser irradiation of an in vitro hydrated type I collagen film containing subsurface chromophores positioned at a given depth. A modified Levenberg-Marquardt nonlinear least-squares algorithm is used to estimate the unknown parameters (i.e., $\left.\chi, C, x_{0}, y_{0}\right)$ for each independent pair of infrared emission images.

An in vitro model using thin (i.e., 50 to $150 \mu \mathrm{m}$ ) type I hydrated collagen films (F1310, Collatec, Plainsboro, NJ) containing variable amounts of absorber was used to simulate discrete chromophores buried in multilayered composite human skin. Chromophores were constructed by staining a film with triphenylmethane dye (Aldrich Chemical Co., Milwaukee, WI), which absorbs optimally at the wavelength utilized in the experiments. Discrete chromophores were prepared by cutting a stained collagen film $\left(w_{z}=125 \mu \mathrm{m}\right)$ with known optical absorption $\left(\mu_{a}=40 \mathrm{~mm}^{-1}\right)$ into a number of thin strips $\left(w_{x}=100\right.$ to $\left.300 \mu \mathrm{m}\right)$. A model skin phantom was constructed by positioning variably spaced (50 to $700 \mu \mathrm{m})$ absorbing thin strips underneath a known thickness $(110 \mu \mathrm{m})$ of a nonabsorbing collagen film. Absorbing thin strips and nonabsorbing films were positioned on a $10-\mathrm{mm}$ thick collagen sponge to simulate an infinite half-space as in in vivo skin (Figure 2).

Pulsed $\left(t_{p}=0.45 \mathrm{~ms}\right)$ radiation $(\lambda=585 \mathrm{~nm})$ released from a flashlamp-pumped dye laser (Model 


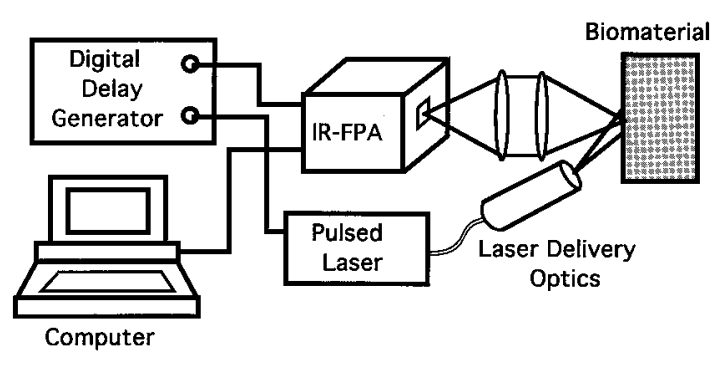

Fig. 3 Schematic diagram of instrumentation to record sequence of infrared emission images $[\Delta M(x, y, t)]$.

SPTL-1, Candela, Wayland MA) was incident on the collagen at 30 degrees from the surface normal and covered a $30-\mathrm{mm}^{2}$ elliptical area. Immediately following exposure to a 0.4-J laser pulse, a time sequence of 35 images of radiometric temperature $[M(x, y, t)]$ was recorded by the IR-FPA camera. Peak radiometric temperature increases at the surface of the in vitro hydrated collagen film never exceeded $7^{\circ} \mathrm{C}$.

A three-element infrared lens (f/2, 50-mm focal length) imaged radiometric temperature $[R(x, y, t)]$ onto a $128 \times 128 \mathrm{InSb}$ IR-FPA (Amber Engineering, Goleta, CA). The collagen was positioned $190 \mathrm{~mm}$ from the infrared imaging lens, giving an image-toobject linear magnification of 0.4 . Efforts to increase spatial resolution by repositioning the lens resulted in unacceptable lens aberrations and large darkfield radiometric temperature variations due to vignetting. The IR-FPA camera acquired 217 images of radiometric temperature per second and was externally triggered by a digital delay generator optically triggered by a fast silicon photo receiver. The infrared signal collected by each detector element in the IR-FPA was digitized with a $3.5-\mathrm{MHz} 12$-bit $(0-4,095)$ A/D converter, immediately stored in random access memory, and later downloaded to a magneto-optic disk storage device (Figure 3). A bandpass infrared filter ( 3 to $5 \mu \mathrm{m}$ ) was positioned near the cold stop of the IR-FPA to reduce background fluctuations and hence increase the signalto-noise ratio (SNR) [Eq. (18)],

$$
S N R=\frac{\overline{\Delta M}}{N E \Delta T},
$$

where $\overline{\Delta M}$ is defined as the mean value of the infrared emission image due to laser heated subsurface chromophores and $N E \Delta T$ is the noise equivalent temperature difference. ${ }^{3}$ Infrared emission images $[\Delta M(x, y, t)]$ were calibrated using a resistor-heated aluminum surface coated with highly emissive $(\epsilon \approx 0.967)$ black paint (TC-303 black, GIE-Tracor, Provo, UT) positioned in object space conjugate to the IR-FPA. A surface-mounted thermistor (Model \#8681, Keithley, Cleveland, $\mathrm{OH}$ ) measured temperature (sensitivity $<0.01^{\circ} \mathrm{C}$ ) as the
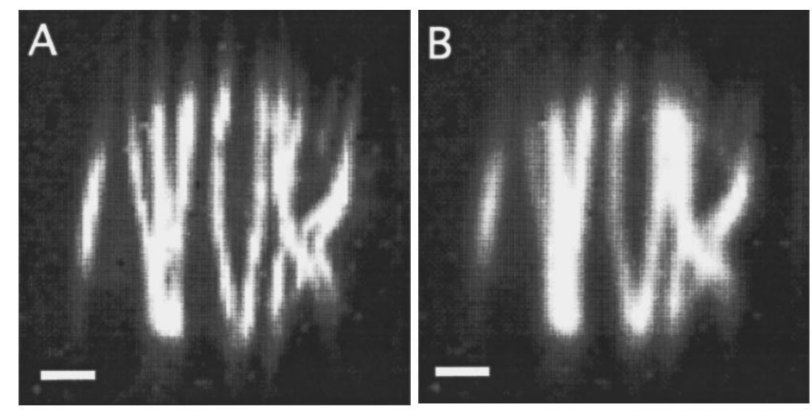

Fig. 4 Infrared emission images recorded at (A) $t_{1}=4.6 \mathrm{~ms}$, and (B) $t_{2}=69 \mathrm{~ms}$. Thermal diffusivity determined from the illustrated image pair is $\chi=0.106 \pm 0.001\left(\mathrm{~mm}^{2} / \mathrm{s}\right)$. Bar represents $1 \mathrm{~mm}$.

surface was slowly heated $\left(1^{\circ} \mathrm{C} / \mathrm{min}\right)$. Lateral spatial resolution was calibrated from an infrared emission image recorded following pulsed laser irradiation of a thin pigmented measuring scale. Strong infrared emission from laser-heated pigment demarcating millimeter increments on the scale allowed accurate determination of the lateral resolution of each pixel in the IR-FPA $(0.14 \mathrm{~mm})$.

Infrared emission image pairs $\left[\Delta M\left(x, y, t_{1}\right)\right.$ and $\left.\Delta M\left(x, y, t_{2}=t_{1}+\Delta t\right)\right]$ separated by at least five frames (i.e., $\Delta t \geqslant 23 \mathrm{~ms}$ ) were used to compute each unknown parameter (i.e., $\chi, C, x_{0}, y_{0}$ ) of the lateral thermal point spread function $\left[K_{r}(\Delta t)\right]$ by application of the modified Levenberg-Marquardt nonlinear least-squares algorithm. ${ }^{4}$ Estimates of the standard deviation of each unknown parameter $\left(\sigma_{\chi}, \sigma_{C}, \sigma_{x}, \sigma_{y}\right)$ deduced from a pair of infrared emission images were determined by a method described by Gallant. ${ }^{5}$ Two recorded sequences of 35 infrared emission images of the in vitro hydrated collagen film allowed computation of 60 independent values of thermal diffusivity $\left(\chi_{i}\right)$. For example, application of the modified LevenbergMarquardt nonlinear least-squares algorithm to infrared emission image pairs (Figure 4) separated by $\Delta t=65 \mathrm{~ms}$, gave $\chi=0.106 \pm 0.001\left(\mathrm{~mm}^{2} / \mathrm{s}\right)$. By invoking the principle of maximum likelihood, ${ }^{6}$ mean thermal diffusivity $(\bar{\chi})$ deduced from all pairs of recorded infrared emission images was computed by a variance-weighted $\left(1 / \sigma_{\chi_{i}}^{2}\right)$ average [Eq. (19)]

$$
\bar{\chi}=\frac{\sum_{i} \chi_{i} / \sigma_{\chi_{i}}^{2}}{\sum_{i} 1 / \sigma_{\chi_{i}}^{2}} .
$$

Standard deviation of the mean $\left(\sigma_{\bar{\chi}}\right)$ was determined [Eq. (20)] from the sum of variances according to

$$
\sigma_{\bar{\chi}}=\left[\frac{1}{N-1} \sum_{i}\left(\chi_{i}-\bar{\chi}\right)^{2}\right]^{1 / 2} .
$$

The mean thermal diffusivity of type I hydrated 
collagen films at room temperature $\left(22^{\circ} \mathrm{C}\right)$ deduced from 60 recorded infrared emission image pairs is $\bar{\chi}=0.103 \pm 0.007\left(\mathrm{~mm}^{2} / \mathrm{s}\right)$.

\section{DISCUSSION AND CONCLUSIONS}

The mean thermal diffusivity of type I hydrated collagen is slightly less than the values for human skin $\left[0.11\left(\mathrm{~mm}^{2} / \mathrm{s}\right)\right] .{ }^{7}$ Due to the presence of protein, the value of thermal diffusivity in collagenous biomaterials is reduced from that of pure water [0.146 $\left.\left(\mathrm{mm}^{2} / \mathrm{s}\right)\right]$ at an equivalent temperature. The water mass content of type I hydrated collagen films used in our experiments is $50 \%$; in contrast, the fractional water mass in human skin, excluding the stratum corneum, is $70 \%{ }^{8}$ We expect and our experiments confirm, therefore, that the thermal diffusivity of type I hydrated collagen is slightly less than that of human skin due to a greater fractional protein content.

The determination of thermal diffusivity using infrared imaging radiometry can be substantially improved and applied to a number of diverse biomaterials. The development of optimal thermal excitation and temporal sampling strategies will give measurement results of higher accuracy with greater computational economy. For example, although the treatment of heat transfer by blood perfusion in Eq. (1) may be invalid in some tissues such as human skin, the method presented to determine $\chi$ is accurate when $\Delta t \ll Q^{-1}$. In human skin, $Q^{-1} \approx 10$ to 60 (s), and thus infrared emission image pairs recorded at times separated by up to $\Delta t=1$ (s) may be used to determine $\chi$. Inasmuch as many previous measurement techniques utilized invasive or semi-invasive probes, spatial resolution has been restricted by practical considerations, and accurate values of in vivo thermal diffusivity are not known. In contrast, infrared imaging radiometry may allow an accurate, high spatial resolution, noncontact determination of thermal diffusivity of many biomaterials. Experiments that determine $\chi$ of various biomaterials are under way in our laboratory and will be reported in the future.

\section{Acknowledgments}

This project was supported by research grants to JSN awarded by the Biomedical Research Technol- ogy Program (R03-RR06988) and Institute of Arthritis and Musculoskeletal and Skin Diseases (1R29AR41638-01A1 and 1R01-AR42437-01A1) at the National Institutes of Health, Whitaker Foundation, and Dermatology Foundation. The work of TEM is supported in part by the Whitaker Foundation. Institutional support from the Office of Naval Research, Department of Energy, National Institutes of Health, and Beckman Laser Institute and Medical Clinic Endowment is also gratefully acknowledged. The work of Dennis Goodman was performed under the auspices of a U. S. Department of Energy grant to the Lawrence Livermore National Laboratory under contract W-7405-Eng-48. The work of B. $\mathrm{S}$. Tanenbaum was supported in part by the National Institutes of Health (R-15-AR43403-01) and a Whitaker Foundation Special Opportunity Award. Sun Microsystems Computing Corporation provided the resources necessary to complete the computations. The Office of Academic Computing at the University of California, Irvine, provided indispensible technical assistance. Chris Johnston of Amber Engineering generously provided the IR-FPA camera system used in the experiments.

\section{REFERENCES}

1. H. F. Bowman, E. G. Cravalho, and M. Woods, "Theory, measurement and application of thermal properties of biomaterials," Ann. Rev. Biophys. Bioeng. 4, 43-80 (1975).

2. W. P. Hansen, T. A. Balasubramaniam, R. J. Greenwald, and H. F. Bowman, "A system for noninvasive thermal conductivity measurements," in Advances in Thermal Conductivity. 13th Int. Conf. Thermal Conductivity, Rolla (1973).

3. G. S. Hopper, "Forward looking infrared systems," in Passive Electro-Optical Systems, S. B. Campana, Ed., Ann ArborBellingham: ERIM-SPIE (1993).

4. J. J. More, "The Levenberg-Marquardt algorithm: implementation and theory," in Numerical Analysis, G. A. Watson, Ed., Lecture Notes in Mathematics, p. 630, Springer-Verlag, Berlin (1977).

5. A. R. Gallant, "Nonlinear regression," Am. Statistician 29, 73-81 (1975).

6. B. R. Frieden, Probability, Statistical Optics, and Data Testing, Springer Verlag, New York (1991).

7. F. A. Duck, Physical Properties of Tissue. A Comprehensive Reference Book, Academic Press, London (1990).

8. T. von Zglinicki, M. Lindberg, G. M. Roomans, and B. Forslind, "Water and ion distribution profiles in human skin," Acta Derm. Venereol. 73, 340-343 (1993).

9. A. M. Patterson, "Modelling the thermal effects of blood flow in human skin," South African Mech. Eng. 28, 179-182 (1978). 